\title{
Vertebral sarcoidosis mimicking metastasis on Magnetic resonance Imaging
}

\author{
Amandeep Singh, Aaina Devgan, Tanveen Kaur, Gauravdeep Singh \\ From Professor, Department of Radiodiagnosis and Imaging, Sri Guru Ramdas Institute of Medical Sciences and Research, Amritsar, Punjab, India
}

\begin{abstract}
Vertebral sarcoidosis is a rare condition that can present with persistent back pain, often with concurrent lung, lymph node, or skin involvement. It can produce lytic or blastic osseous lesions that are indistinguishable from metastatic cancer on bone scan and magnetic resonance imaging (MRI). Here, we present the case of a 65-year-old woman who presented with persistent severe low back pain which progressed gradually and was refractory to usual medical treatment after spontaneous resolution of Stage I pulmonary sarcoidosis. MRI of the spine showed lytic thoracic and lumbar vertebral lesions.
\end{abstract}

Key words: Lytic lesions, Magnetic resonance imaging, Spine, Vertebral sarcoidosis

$\mathrm{S}$ arcoidosis is a multisystem disorder characterized by noncaseating granulomatous infiltration. The most common sites of involvement are the lungs and lymph nodes, while other organs such as the spleen, liver, skin, eyes, muscles, bones, central nervous system (CNS), and salivary glands are less frequently involved [1]. The diagnosis of sarcoidosis is made by a combination of clinical, radiological, and histological findings. Sarcoidosis most frequently involves the lungs, skin, and lymph nodes. CNS involvement is uncommon, occurring symptomatically in $5-15 \%$ of sarcoidosis patients and in about $25 \%$ of patients with systemic sarcoidosis on autopsy studies [2].

Osseous involvement is relatively uncommon in sarcoidosis. The incidence varies from $1 \%$ to $13 \%$ [3]. Most cases of osseous sarcoidosis occur in the long bones of the hands and feet [4]. Vertebral involvement in sarcoidosis is exceedingly rare with $<30$ cases reported [5]. However, histological proof with non-caseating granulomata remains the hallmark of this disease. The diagnosis is sometimes difficult to establish, because sarcoidosis may simulate many diseases, including even metastatic malignancies. Here, we present the case of a 65 -year-old woman with lesions in the vertebral bones, mimicking the presence of extensive metastatic disease, and was presented for magnetic resonance imaging (MRI).

\section{CASE REPORT}

A 65-year-old woman presented with complaints of lower lumbar pain and pain in bilateral legs for 6 months and episodes

\section{Access this article online}

Received - 02 March 2021

Initial Review - 20 March 2021

Accepted - 20 April 2021

DOI: $10.32677 /$ IJCR.2021.v07.i04.014 of whole-body stiffening. Physical examination of the patient showed a well-developed, well-nourished female. The blood pressure, pulse, temperature, and respiratory rate were normal. On local examination, positive findings were confined to the back. Flexion was limited with pain and spasm in the mid-lumbar area.

Laboratory examinations such as hematocrit determination, white blood count, serum calcium, phosphorus, alkaline phosphatase, latex fixation, and serology were normal. The serum electrophoretic pattern showed a moderate increase in the gamma portion. Tuberculin and histoplasmin skin tests were negative.

MRI revealed well-defined areas of altered marrow signal intensity appearing hypointense on T1W and T2W images (Fig. 1) and hyperintense on short TI inversion recovery (STIR) images (Fig. 2) in D11, L1, L2, and S1 vertebral bodies which vary in size from $8-15 \mathrm{~mm}$ in diameter. No obvious loss of vertebral height was noted. On the basis of these findings, a diagnosis of vertebral sarcoidosis was given. On subsequent follow-up, it was found that the patient is being treated on the lines of vertebral sarcoidosis with corticosteroids.

\section{DISCUSSION}

Sarcoidosis is a multisystem disorder characterized by noncaseating granulomatous infiltration. In 2003, Haluska et al. presented a similar patient who was presumed to have widespread metastatic melanoma; vertebral MRI lesions suggested metastatic neoplasm. However, these lesions turned out to be non-necrotizing

Correspondence to: Dr. Amandeep Singh, Department of Radiodiagnosis and Imaging, Sri Guru Ramdas Institute of Medical Sciences and Research, Amritsar, Punjab - 143 001, India. E-mail: dr.amancs@gmail.com

(C) 2021 Creative Commons Attribution-NonCommercial 4.0 International License (CC BY-NC-ND 4.0). 


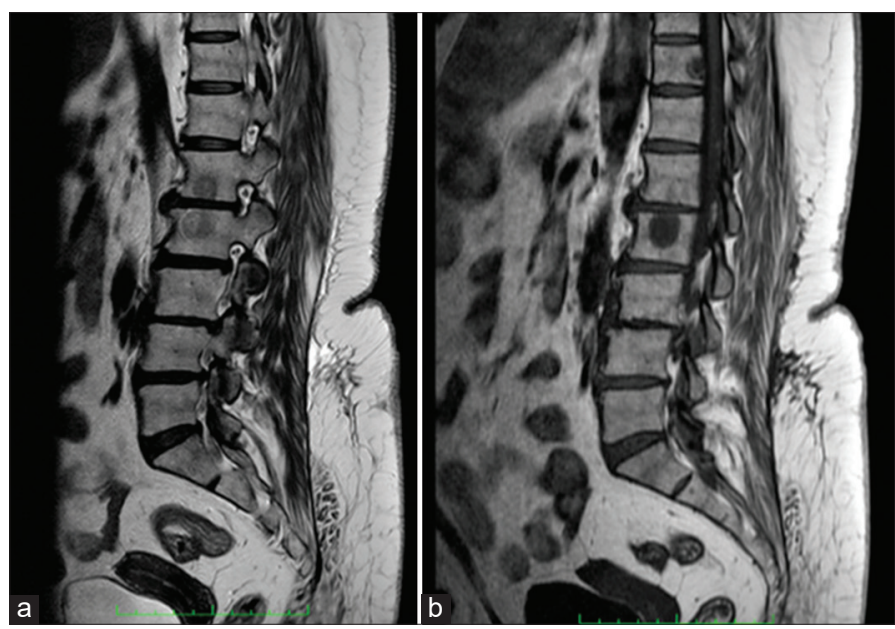

Figure 1: (a) T1W and (b) T2W sagittal magnetic resonance imaging image of the lumbar spine depicting hypointense lesions in (a) L1 and $L 2$ and (b) D11 and $L 2$ vertebral bodies

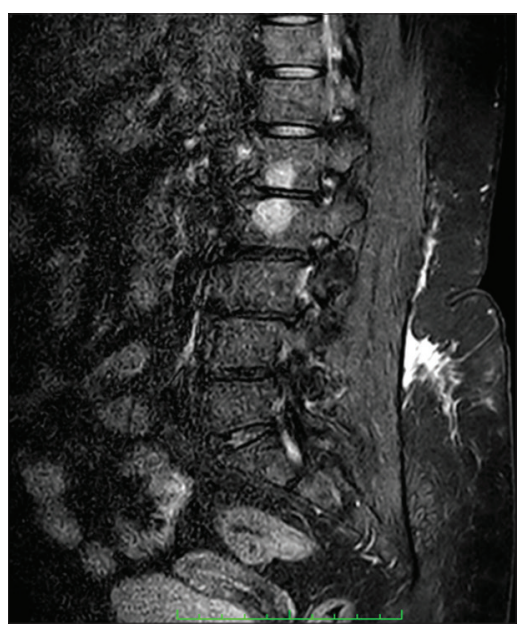

Figure 2: Short TI inversion recovery sagittal magnetic resonance imaging image of the lumbar spine depicting hyperintense lesions in $\mathrm{L} 1$ and $\mathrm{L} 2$ vertebral bodies

granulomas consistent with sarcoidosis [6]. Ludwig et al. reported a patient presenting with low back pain, who was also thought to have metastatic skeletal disease based on MRI, bone scintigraphy, chest computed tomography, and fluorodeoxyglucose (FDG)positron emission tomography (FDG-PET) imaging, which also turned out to be sarcoidosis [3]. A similar case was also reported by Mangino et al. [7].

Vertebral sarcoidosis should be distinguished from spinal cord sarcoidosis. The former affects primarily the vertebral bones, while the latter involves the spinal cord and is classified as neurosarcoidosis. Concurrent vertebral and spinal cord sarcoid are rarely found.

Almost always, the patients with vertebral body sarcoid are symptomatic and present with radicular back pain both during activity and at rest. Tenderness over the involved area may be present. All areas of the vertebral column can be involved, but the thoracic spine is most common. Multiple vertebral bodies are usually affected, and plain radiography can be normal or reveal lytic lesions with or without a rim of sclerosis. An associated paraspinal mass may be present with variable involvement of the pedicle. The disc space can be preserved. Bone scans are non-specific showing uptake by involved bone [7].

Bone scintigraphy has been reported rarely in vertebral sarcoidosis. Although non-specific, it may be a sensitive indicator of the extent of osseous sarcoidosis and has potential diagnostic utility as it can localize sites for biopsy if the clinical area is not readily accessible. In a few cases, MRI findings in vertebral sarcoidosis have been reported. MRI usually demonstrates multifocal lesions within the vertebrae that are hypointense (low signal intensity) on T1-weighted images and hyperintense (high signal intensity) on T2-weighted images, which enhance following contrast medium administration.

Multifocal vertebral body lesions have a broad differential diagnosis that typically includes metastatic disease (in particular prostate, breast, and lung), lymphoma, myeloma, Paget's disease, osteomyelitis, renal osteodystrophy, and granulomatous diseases, which stress the need for further investigation. The rarity of osseous and in particular vertebral sarcoidosis plus its nonspecific imaging manifestations often leads to a significant delay in diagnosis [8]. In our case, a 65-year-old woman presented with lower lumbar pain and pain in bilateral legs for 6 months and episodes of whole-body stiffening. MRI revealed well-defined areas of altered marrow signal intensity appearing hypointense on T1W and T2W images and hyperintense on STIR images in D11, L1, L2, and S1 vertebral bodies which vary in size from 8 $\mathrm{mm}$ to $15 \mathrm{~mm}$ in diameter. No obvious loss of vertebral height was noted.

\section{CONCLUSION}

Osseous and in particular vertebral sarcoidosis is exceedingly rare and a difficult diagnosis to establish because of the resemblance to other diseases, including metastatic malignancy. In our known case of pulmonary sarcoidosis, due to multiple lytic lesions without affecting the vertebral body height, we have given the possibility of vertebral sarcoidosis. On subsequent follow-up, we have found that the patient is being treated on the lines of vertebral sarcoidosis with corticosteroids.

\section{REFERENCES}

1. Fritz D, van de Beek D, Brouwer MC. Clinical features, treatment and outcome in neurosarcoidosis: Systematic review and meta-analysis. BMC Neurol 2016;16:220.

2. Valencia MP, Deaver PM, Mammarappallil MC. Sarcoidosis of the thoracic and lumbar vertebrae, mimicking metastasis or multifocal osteomyelitis by MRI: Case report. Clin Imaging 2009;33:478-81.

3. Ludwig V, Fordice S, Lamar R, Martin WH, Delbeke D. Unsuspected skeletal sarcoidosis mimicking metastatic disease on FDG positron emission tomography and bone scintigraphy. Clin Nucl Med 2003;28:176-9.

4. Jelinek JS, Mark AS, Barth WF. Sclerotic lesions of the cervical spine in sarcoidosis. Skeletal Radiol 1998;27:702-4.

5. Varron L, Broussolle C, Candessanche JP, Marignier R, Rousset H, 
Ninet J, et al. Spinal cord sarcoidosis: Report of seven cases. Eur J Neurol 2009;16:289-96.

6. Haluska P, Luetmer PH, Inwards CY, Afessa B, Shives TC, Ingle JN. Complications of therapy and a diagnostic dilemma case. Case 3. Diagnostic dilemma: Sarcoidosis simulating metastatic malignancy. J Clin Oncol 2003;21:4653-54

7. Mangino D, Stover DE. Sarcoidosis presenting as metastatic bony disease. A case report and review of the literature on vertebral body sarcoidosis. Respiration 2004;71:292-4

8. Scott TF, Yandora K, Valeri A, Chieffe C, Schramke C. Aggressive therapy for neurosarcoidosis: Long-term follow-up of 48 treated patients. Arch Neurol 2007;64:691-6.

Funding: None; Conflicts of Interest: None Stated.

How to cite this article: Singh A, Devgan A, Kaur T, Singh G. Vertebral sarcoidosis mimicking metastasis on Magnetic resonance Imaging. Indian J Case Reports. 2021;7(4):170-172. 